\title{
Predição da biomassa aérea em plantações de Pinus taeda L. por meio de dados LiDAR aerotransportado
}

\author{
Predicting aboveground biomass in Pinus taeda L. \\ plantation using airborne LiDAR data
}

\author{
Carlos Alberto Silva1, Carine Klauberg ${ }^{2}$, Ângela Maria Klein Hentz ${ }^{3}$, \\ Samuel de Padua Chaves Carvalho ${ }^{4}$ e Ana Paula Dalla Corte ${ }^{5}$
}

\begin{abstract}
Resumo
Este trabalho teve como objetivo predizer a biomassa acima do solo (AGB) em plantações de Pinus taeda L., localizados na região sul do Brasil. A base de dados utilizada no estudo foi originada de levantamentos a laser aerotransportados (LiDAR), complementados por informações de campo. Os modelos preditores da biomassa foram ajustados por modelagem não paramétrica, Random Forests (RF), implementado no ambiente R. Para compor os dados de campo foram inventariadas 50 parcelas de área fixa, nas quais foram mensurados os diâmetros de todas as árvores e parte das alturas. Posteriormente o volume individual foi estimado por modelos polinomiais de quinto grau e serviu de base para o cálculo dos valores de AGB. O modelo final, preditor da biomassa, foi composto pelas métricas LiDAR referente a Altura no percentil 99 (H99TH), Coeficiente de Variação da Altura (HCV) e Assimetria da altura (HSKEW) por proporcionarem baixa correlação entre si, e fornecerem os maiores valores de Razão de Melhoria do Modelo (MIR). O modelo final apresentou um o coeficiente de determinação ajustado $\left(R^{2}{ }_{\mathrm{aj}}\right)$ de 0,98 , Raiz Quadrada do Erro Médio (RMSE) de 5,98\%, enquanto que para a validação, esses valores foram de 0,93 e 12,64\%, respectivamente. Portanto conclui-se que o modelo gerou resultados satisfatórios na predição da biomassa aérea em plantações de $P$. taeda, podendo ser considerado como uma ferramenta eficaz no manejo de florestas plantadas.
\end{abstract}

Palavras-chave: Inventário Florestal, Modelagem Não-paramétrica, Plantações Florestais, Métricas LiDAR.

\section{Abstract}

The aim of this study was to predict aboveground biomass (AGB) from Pinus taeda L. plantations, located in South of Brazil, using LiDAR data, in-situ measurements and Random Forests (RF) modeling. Fifty regular sample plots were used, in which the diameter at the breast height (DBH) for all trees and about $15 \%$ of the heights were measured. Afterwards. forest stem volume was predicted using a fifth degree polynomial model, and used to calculate the field AGB values. To create the RF model we selected the H99TH, HCV and HSKEW LiDAR metrics, because they were not highly correlated to each other and presented the higher calculated value of Model Improvement Ratio (MIR). The estimative model of AGB presented a coefficient of determination (Adj. $R^{2}$ ) of 0.98 and RMSE of $5.98 \%$, while for the validation these values were 0.93 and $12.64 \%$, respectively. It was possible to conclude that the RF and LiDAR-derived metrics were able to predict precisely the values of AGB in $P$. taeda plantation, therefore, it can be used as a helpful tool to forest management.

Keywords: Forest inventory, Nonparametric models, Random Forests, LiDAR metrics.

\footnotetext{
${ }^{1}$ Doutorando em Recursos Naturais no Departamento de Recursos Naturais e Sociedade. UI - University of Idaho / College of Natural Resources. 875 Perimeter Drive 83843 - Moscow, ID, USA. E-mail: carlos engflorestal@outlook.com

2Pesquisador Doutor no Departamento de Serviços Florestais. USDA - United States Departament of Agriculture. Rocky Mountain Research Station, 1221 South Main Street, 83843 - Moscow, ID, USA. E-mail: carine klauberg@hotmail.com

32Doutoranda em Engenharia Florestal. UFPR -Universidade Federal do Paraná. Rua Lothário Meissner, 632 - Jardim Botânico - 80210-170 - Curitiba, PR, Brasil. E-mail: elakhentz@gmail.com

${ }^{4}$ Professor Adjunto no Departamento de Ciências Florestais. UFMG - Universidade Federal de Mato Grosso. Av. Fernando Correa da Costa, 2367 - Boa Esperança - 78060900 - Cuiabá, MT, Brasil. E-mail: sam.padua@gmail.com

${ }^{5}$ Professor Adjunto no Departamento de Ciências Florestais. UFPR -Universidade Federal do Paraná. Rua Lothário Meissner, 632 - Jardim Botânico - 80210-170 - Curitiba, PR, Brasil. E-mail: aulacorte@gmail.com
}

Sci. For., Piracicaba, v. 45, n. 115, p. 527-539, set. 2017 DOI: dx.doi.org/10.18671/scifor.v45n115.10 
Silva et al. - Predição da biomassa aérea em plantações de Pinus taeda L. por meio de dados LiDAR aerotransportado

\section{INTRODUÇÃO}

O setor florestal brasileiro tem apresentado grande potencial nos últimos anos, e a silvicultura destaca-se com uma produção aproximada de 195 milhões de toneladas de madeira em 2014, da qual aproximadamente $41 \%$ destinam-se a produção de papel e celulose (IBGE, 2014). De toda essa produção, cerca de 20,54\% das áreas plantadas no Brasil são do gênero Pinus, e na região sul, essa proporção é mais significativa girando em torno de 65\% (IBGE, 2014).

O fornecimento de matéria prima florestal por meio de plantações não é só importante do ponto de vista social e econômico, mas também pelo potencial dessas florestas para sequestro e estoque de carbono atmosférico. As florestas plantadas no Brasil apresentam-se nesse cenário com destaque especial devido ao seu crescimento acelerado (WATZLAWICK et al., 2012), principalmente dos gêneros Pinus e Eucalyptus, sendo que para o primeiro estima-se um teor de carbono de aproximadamente 44,5\% da biomassa seca acima do solo (DALLAGNOL et al., 2011). Para avaliar o potencial de fixação e estoque de carbono em uma floresta são realizados inventários florestais quali-quantitativos. Por métodos diretos, as árvores são cortadas e pesadas (SILVEIRA et al, 2010), já os indiretos estimam a biomassa por meio de equações a partir de outras variáveis, como o diâmetro e a altura (SOMOGYI et al., 2007).

Com o avanço das tecnologias de sensoriamento remoto, estudos utilizando valores de reflectância, obtidos por imagens de satélite, têm sido apresentados para estimar a biomassa (WATZLAWICK et al., 2009). Mais recentemente apresenta-se também a tecnologia LiDAR, pela qual é possível obter-se informações não só da superfície da floresta, mas sim de toda a sua estrutura, inclusive do solo e do relevo (GIONGO et al., 2010), permitindo a obtenção de valores de biomassa mesmo em ambientes complexos, como as florestas secundárias (LU et al., 2012).

Isso ocorre porque a tecnologia LiDAR permite a obtenção de diversas variáveis que se correlacionam com a biomassa florestal, como a contagem de indivíduos, alturas, volume e área basal (CARVALHO et al., 2015; LU et al., 2012; SILVA et al., 2014). Diversos estudos são encontrados quanto a predição da biomassa aérea florestal por meio de dados LiDAR, principalmente na América do Norte e Europa (GARCÍA et al., 2010; Hudak et al., 2012; LU et al., 2012). No Brasil encontram-se trabalhos para florestas naturais (GIONGO et al., 2012), plantios de Eucalyptus spp. (CARVALHO et al. 2015; OLIVEIRA et al., 2014; SILVA et al., 2014) e P. taeda (ZANDONÁ et al., 2008).

Apesar do crescente número de publicações sobre o tema LiDAR, os estudos no Brasil ainda são incipientes, logo pesquisas de cunho científico relacionadas principalmente ao custo/benefício, processamento e modelagem são consideradas essenciais. Segundo Lu et al. (2012), os principais problemas de estimativas de biomassa estão diretamente relacionados aos procedimentos de modelagem. Dentre os métodos estatísticos, a modelagem não paramétrica tem ganhado atenção especial na modelagem de parâmetros da estrutura florestal (LATIFI et al., 2010), uma vez que não há a necessidade de atendimento às premissas básicas dos modelos convencionais de regressão como normalidade e homogeneidade de variância dos resíduos, além de garantia na habilidade de modelar relações complexas e com grandes bases de dados (MUTANGA et al., 2012).

Dentre os modelos não paramétricos, destacam-se o algoritmo Random Forests (RF) (LATIFI et al., 2010; NEUMANN et al., 2011). O algoritmo Random Forests foi desenvolvido por Breiman (2001) e baseia-se na construção de diversas árvores de regressão de forma aleatória e independentes (BREIMAN, 2001), cada qual tem um voto, que é utilizado para determinar a classe do atributo avaliado, com base na classe que obteve mais votos (BREIMAN, 2001). Comparando-se com outras abordagens, o algoritmo RF mostra-se superior as tradicionais técnicas de estimativa de biomassa por modelos paramétricos e dados LiDAR, como observado por Cao et al. (2014), Zhao et al. (2012) e Sankey et al. (2013), em diferentes contextos.

Dado o exposto, neste trabalho objetivou-se integrar processos de modelagem não paramétrica à dados originados de sobrevoos LiDAR para a predição da AGB visando a precisão e robustez nas análises sugeridas e aplicadas em plantações de Pinus taeda L. com diferentes idades, no sul do Brasil. 


\section{MATERIAL E MÉTODOS}

\section{Caracterização da área de estudo}

O estudo foi realizado em plantações de Pinus taeda localizados no município de Telêmaco Borba, estado do Paraná (Figura 1) e pertencentes a empresa Klabin S/A. Para a formação das florestas foram utilizados espaçamentos médios iniciais de 3 x $2 \mathrm{~m}$ ou 2,5 x 2,5 m, resultando em uma densidade média de árvores de 1.667 e 2.000 por hectare, respectivamente.
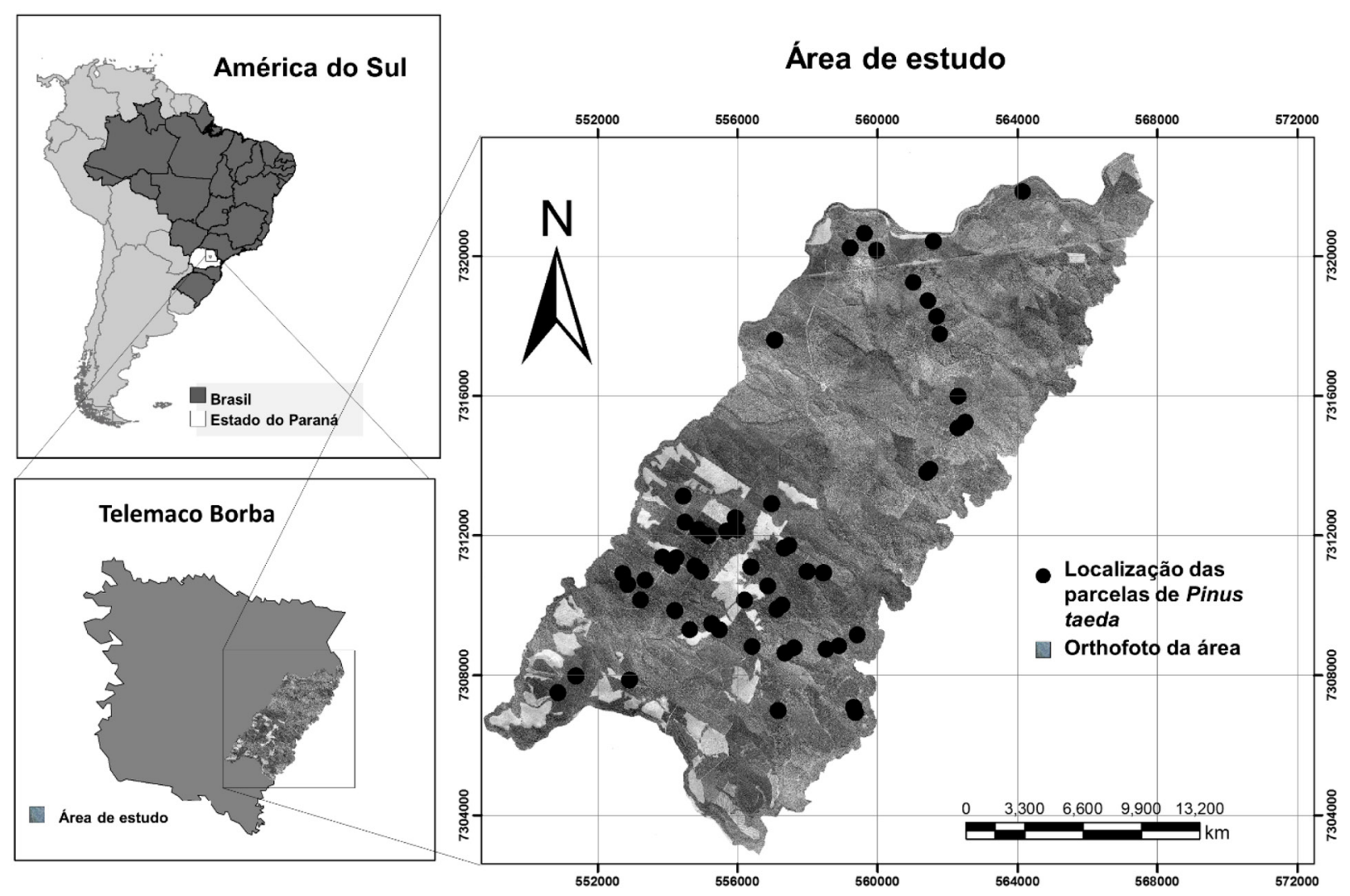

Figura 1. Localização da área de estudo, no estado do Paraná, Brasil. Os pontos indicam a localização das 50 parcelas de campo sobre plantação de $P$. taeda.

Figure 1. Study area located in the State of Paraná, Brazil. The points indicate the location of the field plots in $P$. taeda plantation.

O clima da região é descrito por Köppen e Geiger como Cfa, temperado úmido com verão quente (ALVARES, et al. 2013). A precipitação total anual é de aproximadamente $1.378 \mathrm{~mm}$, e a temperatura média anual de $18,4^{\circ} \mathrm{C}$. A topografia da área de estudo varia de ondulado a montanhoso, com altitude variando de $618 \mathrm{~m}$ à $905 \mathrm{~m}$. Os talhões florestais estão localizados em áreas com topografia relativamente plana, e são geridos pela própria empresa.

\section{Coleta dos dados de campo}

Em campo foram mensurados dados de inventário florestal em 50 parcelas com áreas variando de 500 a $620 \mathrm{~m}^{2}$. A idade da floresta varia de 3 a 9 anos. Todas as parcelas foram georreferenciadas com GPS com capacidade de correção diferencial modelo Trimble Pro-XR, sendo que em cada parcela foram mensurados os diâmetros, medidos a 1,3 m do solo (dap) e $15 \%$ das alturas totais (ht). As alturas das árvores não mensuradas em campo foram preditas por relação hipsométrica. Este procedimento foi efetuado pelo sistema de processamento de dados da própria empresa. Informações do volume médio dos talhões $\left(\mathrm{m}^{3} \cdot \mathrm{ha}^{-1}\right)$ foram também fornecidos pela empresa Klabin(Tabela 1).

Os valores de volume foram calculados utilizando um polinômio de quinto grau de acordo com Schöpfer (1966). Os modelos polinomiais foram ajustados por classe de diâmetro, e os coeficientes desses modelos são propriedade intelectual da empresa. As estatísticas que refletem a qualidade preditiva dos modelos estão apresentadas na Tabela 1. 
Tabela 1. Estatística dos Modelos de afilamento.

Table 1. Taper models statistics.

\begin{tabular}{lcccc}
\hline Amplitude de dap (cm) & $\mathbf{R}^{\mathbf{2}}{ }_{\mathbf{A j} .}$ & $\mathbf{r}$ & \multicolumn{2}{c}{$\mathbf{S}_{\mathbf{y x}} \mathbf{\%}^{\mathbf{d}}$} \\
\hline $0,0-17,9$ & 0,96 & 0,98 & 9,58 & $\mathbf{V}$ \\
$18,0-29,9$ & 0,98 & 0,99 & 7,99 & 9,35 \\
$30,0-70,0$ & 0,98 & 0,99 & 7,52 & 8,21 \\
\hline
\end{tabular}

dap= diâmetro a $\mathrm{I}, 30 \mathrm{~m}$ do solo $(\mathrm{cm}) ; \mathrm{V}=$ volume médio do talhão $\left(\mathrm{m}^{3} \cdot h \mathrm{~h}^{-1}\right)$.

Para a estimativa da biomassa acima do solo (AGB) foi utilizada a Equação 1:

$$
\mathrm{AGB}=\frac{\mathrm{D} * \mathrm{~V}}{1000}
$$

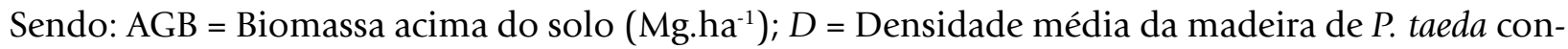
siderada de $394 \mathrm{~kg} \cdot \mathrm{m}^{3}$ (RIGATTO et al., 2004); e V= volume médio do talhão ( $\mathrm{m}^{3}$.ha ${ }^{-1}$ ).

As estatísticas dos estoques de AGB (Mg.ha-1) mensurados em campo estão apresentados na Tabela 2.

Tabela 2. Valores de AGB em campo para $P$. taeda na área de estudo $(n=50)$.

Table 2. Field-based AGB of the $P$. taeda in the study area $(n=50)$.

\begin{tabular}{|c|c|c|c|c|c|}
\hline \multirow{2}{*}{ Idades (anos) } & \multicolumn{4}{|c|}{ AGB (Mg.ha-1) } & \multirow{2}{*}{$\mathbf{N}$} \\
\hline & Min & Max & Média & Sd & \\
\hline $3 \geq 1<5$ & 12,58 & 28,35 & 22,16 & 4,33 & 19 \\
\hline $5 \geq 1<7$ & 29,15 & 78,42 & 52,86 & 12,12 & 18 \\
\hline $7 \geq 1<9$ & 40,58 & 80,01 & 65,82 & 10,70 & 16 \\
\hline Média & 27,44 & 62,26 & 46,95 & 9,05 & 53 \\
\hline
\end{tabular}

Min= Mínima AGB; Max= Máxima AGB; Sd= Desvio padrão; N= número observado.

\section{Aquisição e processamento dos dados LiDAR}

Os dados LiDAR foram obtidos com o sensor Harrier 68i embarcado em uma aeronave CESSNA 206. As características e a precisão dos dados estão apresentadas na Tabela 3. O processamento dos dados LiDAR se deu em 7 etapas conforme Figura 2. Inicialmente a nuvem de pontos LiDAR foi tratada de forma a obter dois principais produtos: o modelo digital do terreno (MDT) e as métricas LiDAR de estrutura da copa. Para o processamento dos dados LiDAR utilizou-se o software FUSION/LDV 3.42, desenvolvido e disponibilizado pelo Serviço Florestal Norte Americano (MCGAUGHEY, 2015).

Tabela 3. Características do voo LiDAR.

Table 3. LiDAR flight characteristics.

\begin{tabular}{lc}
\hline Parâmetros & Valores \\
\hline Ângulo de visada $\left(^{\circ}\right)$ & $60^{\circ}$ \\
Largura da faixa imageada $(\mathrm{m})$ & $0,33 \mathrm{~m}$ \\
Velocidade do voo $(\mathrm{km} / \mathrm{h})$ & $234,00 \mathrm{~km} / \mathrm{h}$ \\
Acurácia Horizontal & $10 \mathrm{~cm}$ \\
Acurácia Vertical & $15 \mathrm{~cm}$ \\
Altitude do voo & $666,17 \mathrm{~m}$ \\
Frequência & $300 \mathrm{kHz}$ \\
Densidade de Pulsos & $4 \mathrm{pulses} / \mathrm{m}^{2}$ \\
\hline
\end{tabular}

Como passo inicial, a função Catalog foi utilizada para gerar um resumo descritivo das informações. Na sequência aplicou-se o algoritmo de filtragem Groundfilter, tomando como referência os trabalhos de Kraus e Pfeifer (1998), de forma a diferenciar pontos no solo, de vegetação. A partir dos pontos classificados como solo foi gerado o MDT, com resolução espacial de um metro, e utilizando a função GridSurfaceCreate. Após essa etapa, a função ClipData foi utilizada para normalizar as alturas. A função PolyClipdata foi utilizada então para recortar a nuvem de pontos LiDAR para as 50 parcelas mensuradas em campo, e a ferramenta Cloudmetrics aplicada para computar as métricas LiDAR em cada uma das 50 parcelas, utilizando todos as retornos da nuvem de pontos. Por fim, a 
função GridMetrics gerou as métricas LiDAR em um grade de células de resolução espacial de 5 metros, em toda a região. Uma listagem das métricas LiDAR estão apresentadas na Tabela 4.

Tabela 4. Métricas derivadas do LiDAR candidatas como variáveis para os modelos de estimativa da AGB (MCGAUGHEY, 2015).

Table 4. LiDAR-derived metrics considered as candidate variables for predictive AGB models (MCGAUGHEY, 2015).

\begin{tabular}{ll}
\hline Métrica & Descrição \\
\hline HMIN & Altura mínima \\
HMAX & Altura máxima \\
HMEAN & Altura medica \\
HMAD & Mediana da altura \\
HSD & Desvio padrão da altura \\
HVAR & Variância da altura \\
HSKEW & Assimetria da altura \\
HKURT & Curtose da altura \\
HCV & Coeficiente de variação da altura \\
HMODE & Moda da altura \\
H05TH & Percentil 5 da altura \\
H10TH & Percentil 10 da altura \\
H20TH & Percentil 20 da altura \\
H25TH & Percentil 25 da altura \\
H30TH & Percentil 30 da altura \\
H40TH & Percentil 40 da altura \\
H50TH & Percentil 50 da altura \\
H60TH & Percentil 60 da altura \\
H70TH & Percentil 70 da altura \\
H75TH & Percentil 75 da altura \\
H80TH & Percentil 80 da altura \\
H90TH & Percentil 90 da altura \\
H95TH & Percentil 95 da altura \\
H99TH & Percentil 99 da altura \\
COV & Cobertura de copa (Porcentagem de primeiro retorno acima de 1.30m) \\
\hline
\end{tabular}

\section{Modelagem}

Os procedimentos computacionais para elaboração do modelo de predição da biomassa foram realizados no software R com o uso do pacote randomForest (BREIMAN, 2001; LIAW; WIENER, 2002). As métricas LiDAR, que compuseram o modelo, foram selecionadas e avaliadas em duas etapas. Primeiro, o Coeficiente de Correlação de Pearson (r) foi calculado e utilizado para identificar variáveis altamente correlacionadas, ou seja, superiores a 0,9 como sugerido por Hudak et al. (2012) e Silva et al. (2014). Se um grupo de métricas (2 ou mais) apresentasse alta correlação, foi mantida apenas uma dessas métricas, e excluídas as demais. Em seguida foram identificadas as métricas mais relevantes, baseando-se no valor de MIR (Razão de Melhoria do Modelo, do inglês Model Improvement Ratio), que calcula uma média de importância de variáveis padronizada (EVANS et al., 2010). Ao todo foram realizadas 1000 iterações do modelo RF com as métricas LiDAR não correlacionadas, e obteve-se o valor de MIR de cada métrica. Por fim, foram utilizadas as métricas com maiores valores de MIR para criar o modelo RF final para a predição da AGB.

A precisão dos modelos foi avaliada pela Percentagem de Variação Explicada pelo modelo (PEV \%), Coeficiente de Determinação Ajustado $\left(\mathrm{R}^{2}{ }_{\mathrm{Aj}}\right)$, Raiz Quadrada do Erro Médio (RMSE) e BIAS, conforme as equações 2 e 3 .

$$
\begin{aligned}
& \text { RMSE }=\sqrt{\frac{\sum_{=1}^{n}\left(y_{i}-\hat{y}_{i}\right)^{2}}{n}} \\
& \text { BIAS }=\frac{1}{n} \sum_{i=1}^{n}\left(y_{i}-\hat{y}_{i}\right)
\end{aligned}
$$

Em que: $n$ é o número de parcelas, $y_{i}$ é o valor observado para a parcela $i$, e $\hat{y}_{i}$ é o valor predito para a parcela $i$. Os valores relativos de RMSE e BIAS foram também calculados com relação à média da AGB observada no campo. O modelo foi definido como aceitável quando apresentou valor de RMSE e BIAS abaixo de 10\%. 
O desempenho preditivo do modelo RF para AGB foi complementado pela análise de validação cruzada "deixa-um de fora" - LOOCV, do inglês leave-one-out cross-validation. Testes de equivalência estatística, descritos por Robinson et al. (2005), foram aplicados para avaliar a similaridade dos valores preditos versus os observados.

Por fim, foi utilizada a função AsciiGridPredict do pacote yaImpute no $R$ (CROOKSTON; FINLEY, 2008) para aplicar o modelo para toda a região, e por consequência, mapear a distribuição espacial da biomassa aérea nas plantações $P$. taeda em nível de Talhão, com resolução espacial de $5 \mathrm{~m}$. Um fluxograma dos procedimentos adotados neste estudo está representado na Figura 2.

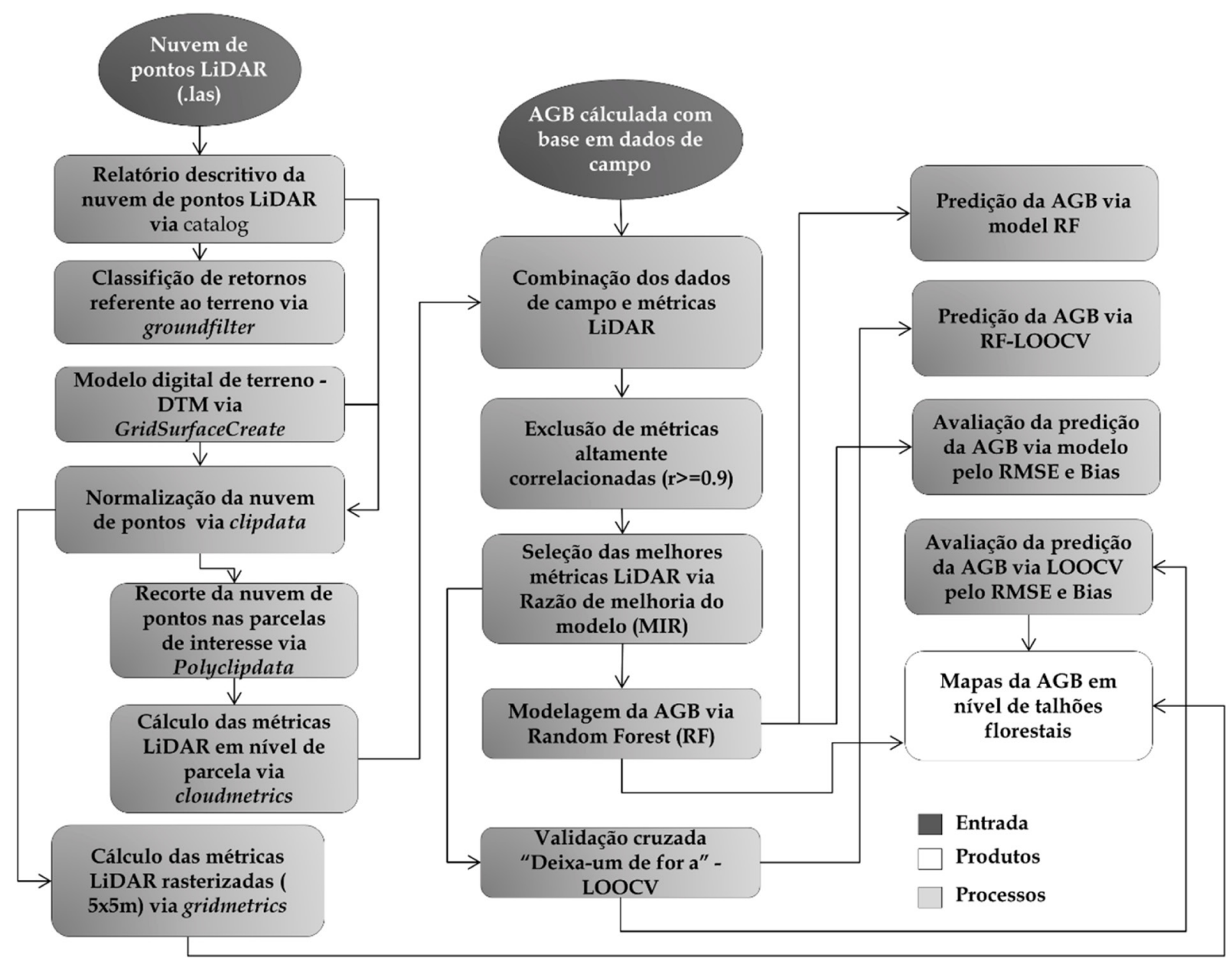

Figura 2. Procedimentos executados para a predição dos valores AGB a partir de dados LiDAR e parcelas inventariadas, em plantio de $P$. taeda.

Figure 2. Procedure for predicting AGB from LiDAR and inventory plot data in P. taeda plantation.

\section{RESULTADOS E DISCUSSÃO}

\section{Seleção das métricas LiDAR}

Para seleção das métricas LiDAR candidatas foi obtido o coeficiente de Correlação de Pearson (r) adotando um nível de exclusão de 0,9. De um total de 25 variáveis candidatas, seis mostraram-se não correlacionadas com as demais, sendo elas: altura mínima (HMIN), variância da altura (HVAR), coeficiente de variação da altura (HCV), assimetria da altura (HSKE), curtose da altura (HKUR) e a covariância da altura (COV). A métrica percentil 99\% (H99TH) da altura foi mantida uma vez que esta foi o ponto de partida para exclusão das demais. Sendo assim um total de sete variáveis foram selecionadas para compor o modelo RF (Tabela 5). 
Tabela 5. Correlação de Pearson entre as métricas LiDAR não altamente correlacionadas.

Table 5. Pearson's correlation among LiDAR metrics not high correlated.

\begin{tabular}{lccccccc}
\hline$r$ & HMIN & HVAR & HCV & HSKE & HKUR & H99TH & COV \\
\hline HMIN & 1.00 & & & & & & \\
HVAR & -0.04 & 1.00 & & & & & \\
HCV & -0.46 & 0.10 & 1.00 & & & & \\
HSKE & -0.31 & -0.18 & 0.84 & 1.00 & & & \\
HKUR & 0.25 & -0.10 & -0.83 & -0.87 & 1.00 & & \\
H99TH & 0.26 & 0.74 & -0.53 & -0.74 & 0.50 & 1.00 & 1.00 \\
COV & 0.21 & 0.21 & -0.69 & -0.66 & 0.49 & 0.54 & \\
\hline
\end{tabular}

Selecionadas as métricas, posteriormente foram efetuadas 1000 iterações do modelo RF para calcular os valores de razão de melhoria do modelo (MIR). Das 7 métricas selecionadas, as 3 mais significativas na resposta da biomassa foram H99TH, HCV e HSKE uma vez em todas as 1000 iterações apresentaram os maiores valores de MIR (Tabela 6). Portanto, essas três métricas foram utilizadas para a construção do modelo final de predição de biomassa pelo RF.

Tabela 6. Resumo dos valores de Razão de Melhoria do Modelo - MIR.

Table 6. Summary of the model improvement ratio - MIR.

\begin{tabular}{lccccccc}
\hline \multirow{2}{*}{ Estatísticas } & Métricas Derivadas do LiDAR \\
\cline { 2 - 8 } & HMIN & HVAR & HCV & HSKE & HKUR & H99TH & COV \\
\hline $\mathrm{n}$ & 0 & 1000 & 1000 & 1000 & 0 & 1000 & 0 \\
MIR & 0 & 0.26 & 0.33 & 0.91 & 0 & 1.00 & 0 \\
\hline
\end{tabular}

$\mathrm{N}=$ número de iterações em que cada métrica foi selecionado; $\mathrm{MIR}=$ valor de Razão de Melhoria do Modelo calculado.

Em geral as métricas de altura derivadas de dados LiDAR apresentam boa correlação com atributos da floresta, e, portanto, são utilizadas em modelos preditivos. Tonolli et al. (2011) destacou as métricas média da altura (HMEAN), o seu valor máximo (HMAX) e o coeficiente de variação (HCV) para estimativa de volume. Diferentes percentis da altura das árvores na contrução de modelos de biomassa e/ou carbono acima do solo a partir de dados LiDAR foram destacados por Sheridan et al. (2015); Silva et al. (2014); Zhao et al. (2012). A métrica de altura média foi apontada como uma das mais significativas para Hyde et al. (2007) e Sheridan et al. (2015) para estimativas de biomassa acima do solo em plantações de Pinus sp.

\section{Ajuste e validação do Modelo de biomassa Random Forest}

O modelo ajustado para estimar os valores de AGB para as parcelas apresentou um valor de Percentagem de Variação Explicada (PVE) de 92,54\%, ou seja, esse modelo consegue explicar mais de $90 \%$ da variação dos estoques de biomassa acima do solo. O modelo para as parcelas apresentou um coeficiente de determinação $\left(\mathrm{R}_{\mathrm{Aj}}^{2}\right)$ de 0,98 , enquanto que para os dados da validação, esse valor foi de 0,93, de forma que o modelo apresentou resultados satisfatórios tanto para as amostras de treinamento quanto para as de validação. Outro ponto relevante foram os baixos valores RMSE e BIAS, conforme Tabela 7, que corroboram na qualidade preditiva do modelo.

Tabela 7. Estatísticas dos modelos ajustados pelo RF para predição da AGB.

Table 7. Statistics of the fitted RF models for AGB prediction.

\begin{tabular}{|c|c|c|c|c|c|c|c|}
\hline \multirow{2}{*}{ RF } & \multirow{2}{*}{ Métricas derivadas do LiDAR } & \multirow{2}{*}{$\mathbf{R}^{2} \mathbf{A j}$} & \multirow{2}{*}{$\mathbf{r}$} & \multicolumn{2}{|c|}{ RMSE } & \multicolumn{2}{|c|}{ Bias } \\
\hline & & & & Mg ha $^{-1}$ & $\%$ & $\mathrm{Mg} \mathrm{ha}^{-1}$ & $\%$ \\
\hline Modelo & \multirow{2}{*}{$\mathrm{H} 99 \mathrm{TH}+\mathrm{HSKE}+\mathrm{HCV}$} & 0.98 & 0.99 & 2.71 & 5.98 & -0.17 & -0.37 \\
\hline LOOCV & & 0.93 & 0.96 & 5.71 & 12.64 & -0.55 & -1.22 \\
\hline
\end{tabular}

Singh et al. (2004) afirmam que RMSE com valores inferiores a metade do desvio padrão dos dados medidos podem ser considerados muito baixos indicando que o modelo é apropriado. Aplicando-se a tese proposta pelos autores para os dados aqui apresentados, ter-se-ia um valor limite de aproximadamente $10 \%$. Os valores a serem comparados seriam de 5,98\% referente ao modelo, que estaria dentro do limite proposto e o valor de $12,64 \%$ referente à validação cruzada, um pouco acima do valor proposto. No entanto, quando comparadas às variações para a variável biomassa em outros trabalhos com coníferas, percebe-se que estes valores estão no limite inferior da variação 
encontrada. Muukkonen e Makipää (2006) em trabalhos com biomassa em florestas reportam valores de RMSE para os modelos desenvolvidos, variando entre 16,6\% e 147,0\%. White et al. (2015) estudando a biomassa de coníferas com a tecnologia LiDAR no Canadá, encontrou RMSE variando entre $15,461 \%$ e 51,784\%, justificando portanto a aceitação dos valores encontrados neste trabalho.

Graficamente (Figura 3) observa-se a similaridade entre os valores observados versus os estimados, tanto para a amostra de treinamento como para as de validação, demonstrando portanto, que os valores preditos e observados são equivalentes, com baixa variabilidade, principalmente para o modelo RF com as amostras de treinamento.

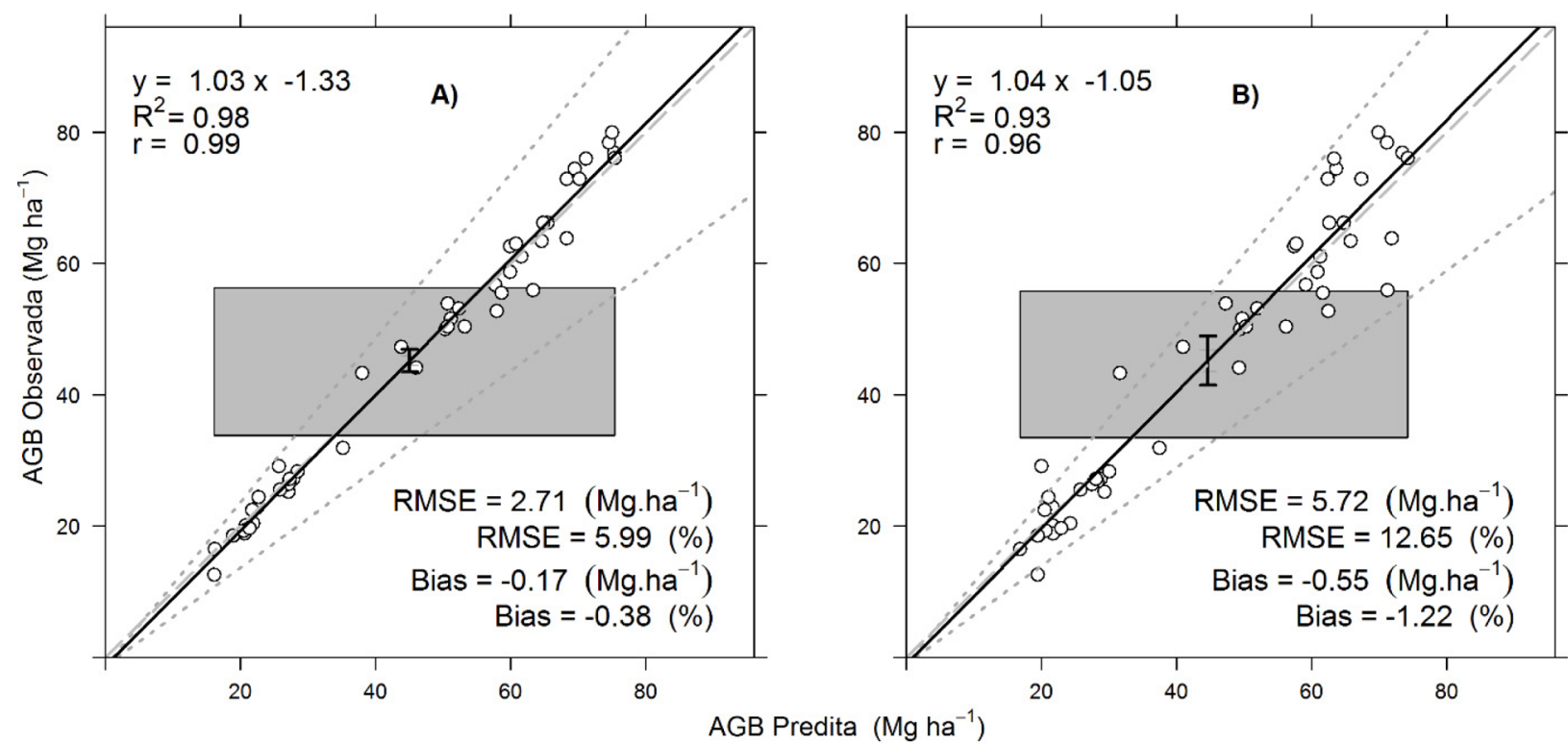

Figura 3. Gráfico de Equivalência entre os valores observados e preditos de AGB, pelo modelo RF de treinamento (A) modelo RF de validação LOOCV (B); $n=50$.

Figure 3. Equivalence plot of the observed versus predicted $A G B$ by the RF-model $(A)$; observed versus predicted AGB by the RF-LOOCV (B); $(n=50)$.

\section{Predição da Biomassa Acima do Solo}

A Figura 4 apresenta a diferença de biomassa entre as distintas classes de idade. É possível verificar que os valores de biomassa são tanto dependentes da idade, quanto do modelo utilizado. Os valores são próximos à $20 \mathrm{Mg} \cdot$ ha $^{-1}$ para a classe entre 3 e 5 anos, $55 \mathrm{Mg}$.ha- ${ }^{1}$ para a classe entre 5 e 7 anos, e $60 \mathrm{Mg} \cdot$ ha- $^{1}$ para a classe entre 7 e 9 anos. A maior variabilidade foi notada na classe entre 5 e 7 anos.

No estudo de Sette Júnior et al. (2006) desenvolvido em Rio Negrinho (SC), em povoamentos de $P$. taeda com diferentes idades ( 5 a 12 anos), observaram que a biomassa arbórea total, correspondente ao tronco da árvore variou de $12,04 \mathrm{Mg} \cdot \mathrm{ha}^{-1}$ aos 5 anos e 147,83 Mg.ha- ${ }^{-1}$ aos 12 anos. Schumacher et al. (2002) para o estoque de biomassa em plantios de P. taeda no Rio Grande do Sul, encontraram 41,3 Mg.ha' ${ }^{-1}$ aos 5 anos de idade, 106,8 Mg.ha' ${ }^{-1}$ aos 10 anos de idade e 277,2 Mg.ha-1 aos 15 anos de idade. Balbinot et al. (2003) estudando a estimativa do carbono orgânico de um plantio de P. taeda L., aos 5 anos de idade, no município de Cambará do Sul, estado do Rio Grande do Sul, constataram valores de biomassa seca total de 41,5 Mg.ha- ${ }^{-1}$, considerando um total de 1.600 árvores por hectare, ou seja, espaçamento $3 \times 2 \mathrm{~m}\left(6,0 \mathrm{~m}^{2}\right)$. Schikowski et al. (2013) observaram em plantios do gênero Pinus, aos 5 anos de idade uma biomassa de 55,373kg por indivíduo, que

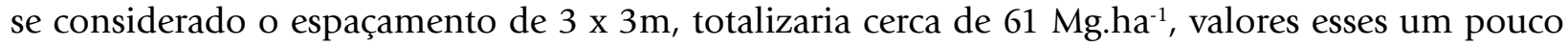
superiores aos encontrados neste trabalho, aos quais são explicados pelas diferenças em qualidade de sítio e principalmente pela diferença em idade. Neste estudo, avaliamos parcelas com idade entre 3 e 9 anos e não possuímos parcelas amostrais representativas de talhões com idade superior a 9 anos, como observado nos trabalhos citados anteriormente. 


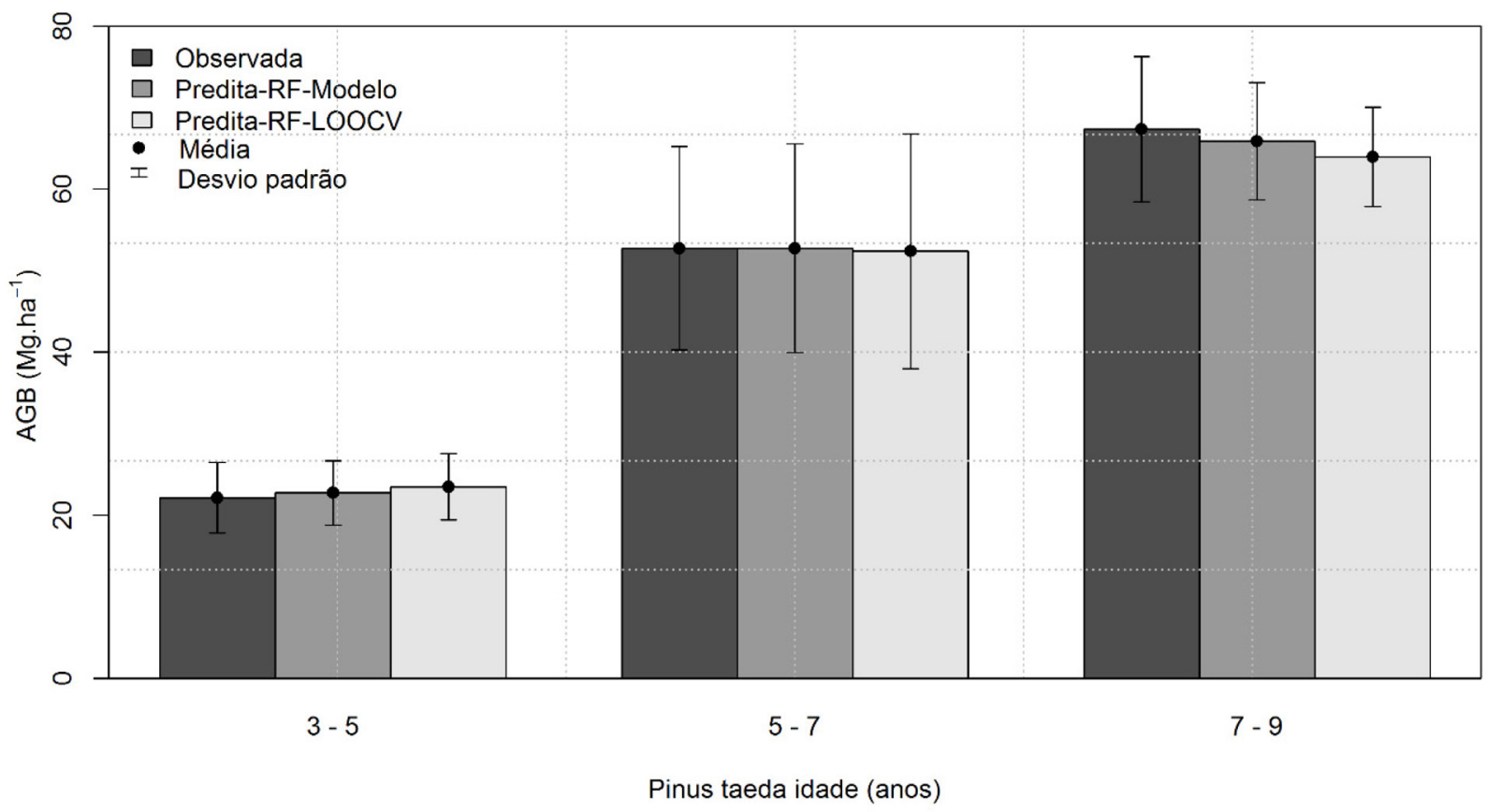

Figura 4. Predição da AGB entre as classes de idade (anos) do plantio.

Figure 4. AGB prediction across plantation age (years).

Na Figura 5, apresentamos os mapas da predição de biomassa para 3 parcelas, cada uma referente à uma das 3 classes de idade. Esses resultados auxiliam a demonstrar o potencial do método como uma nova ferramenta para o manejo florestal, em que é possível identificar variações de biomassa com grande precisão.
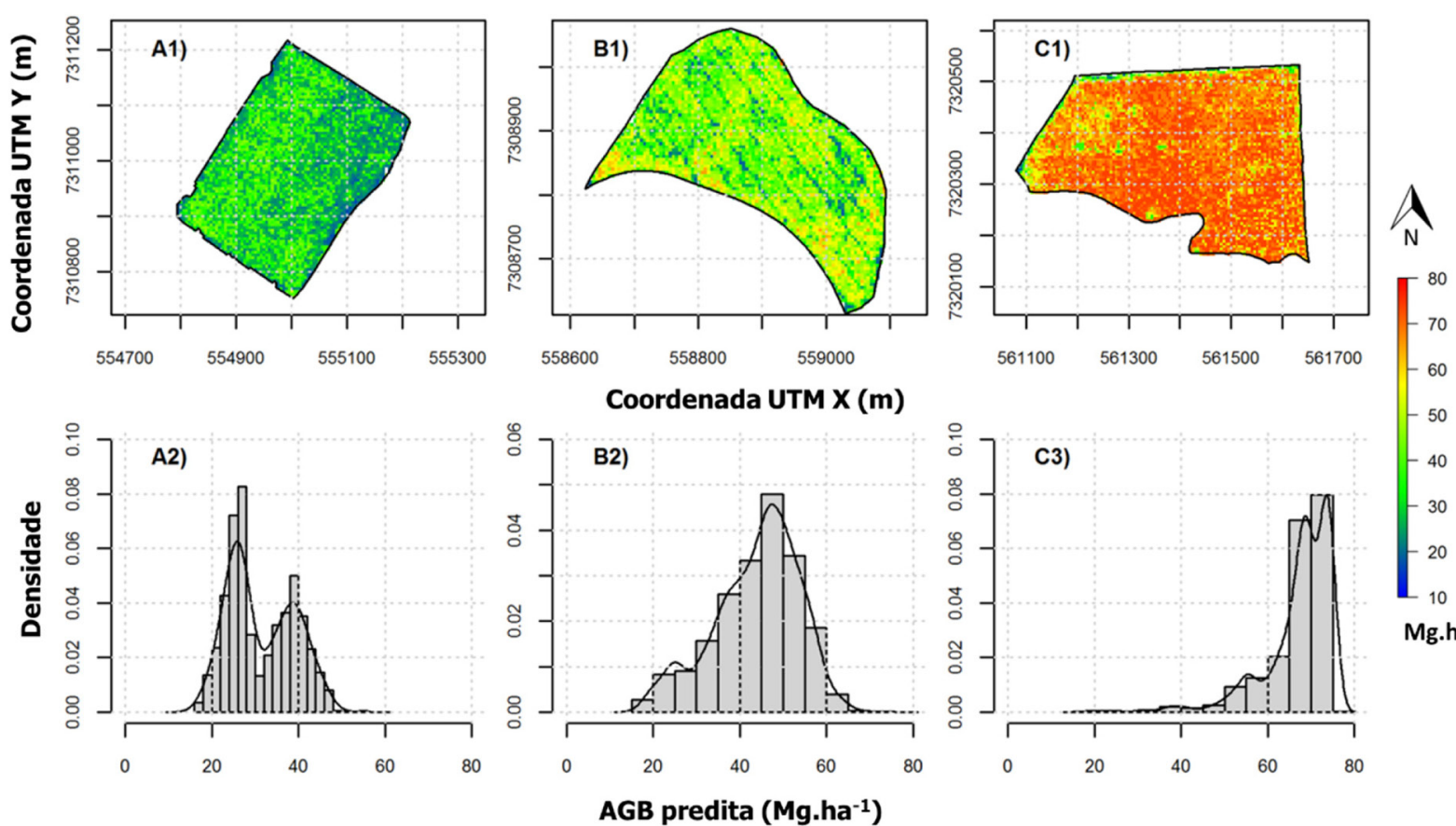

Figura 5. Predição da $A G B$ à nível de talhões. Mapas de predição (1) e densidade de valores de $A G B$ preditos em nível de talhão pelo RF (2). Classes de idade, anos, 3-5 (A), 5-7 (B) e 7-9 (C).

Figure 5. AGB prediction at stand-level. Map prediction (1) and Density plot of the AGB predicted by the RF (2). Ages ranging, in years, 3-5 (A), 5-7 (B) e 7-9 (C).

Na Figura 5 observa-se que os talhões não são totalmente homogêneos, mesmo tratando-se de um plantio equiâneo. No talhão com as menores idades (A2), observa-se que a região nordeste apresenta menor estoque de biomassa que o restante do talhão, hipoteticamente devido às diferenças de sítio. Da mesma forma, no talhão correspondente a idade entre 7 e 9 anos (C2), apesar do 
estoque de biomassa estar entre 70-80Mg.ha-1, em alguns pontos observam-se valores de até 40Mg. $\mathrm{ha}^{-1}$, resultantes das falhas no plantio ou mortalidade de indivíduos.

Destaca-se que as diferenças apontadas podem representar um diferencial significativo no estoque total de biomassa do talhão. Portanto, verificar se há a possibilidade de se produzir mapas de estimativa de biomassa a partir de dados LiDAR, sendo os mesmos de grande valia para uma melhor gestão das indústrias de base florestal.

Além do LiDAR, fonte de dados com grande capacidade em obter a caracterização tridimensional das estruturas complexas de uma floresta (CAO et al., 2014; GIONGO et al., 2010), a modelagem do algoritmo RF mostra-se muito precisa, como observado por Neumann et al. (2011). O autor destaca a utilidade destes modelos na predição de biomassa em extensas áreas. Estes resultados estão associados à estabilidade de pequenas perturbações no conjunto de dados bem como à sua acurácia (CLUTER et al., 2007). Mesmo verificada a sua eficiência, quando utilizado o RF é importante destacar que de acordo com Penner et al. (2013) esta abordagem necessita de uma base de dados adequada visando o treinamento do modelo, e portanto recomenda-se sempre utilizar-se de uma base de dados representativa ao construir o modelo.

\section{CONCLUSÕES}

Conclui-se que a integração dos procedimentos adotados neste estudo apresenta grande potencial em espacializar os estoques de biomassa em talhões de Pinus taeda, o que certamente contribui para uma melhor gestão florestal, uma vez que os valores preditos de AGB estão dentro dos parâmetros de precisão necessária, tanto para o modelo gerado utilizando-se das amostras de treinamento, como pelo modelo da validação cruzada.

\section{AGRADECIMENTOS}

À Klabin S/A pelo fornecimento de dados de inventário e lidar. Assim como aos revisores anônimos pelas valiosas sugestões.

\section{REFERÊNCIAS BIBLIOGRÁFICAS}

ALVARES, C. A.; STAPE, J. L.; SENTELHAS, P. C.; GONÇALVES, J. L. M.; SPAROVEK, G. Köppen's climate classification map for Brazil. Meteorologische Zeitschrift, Berlin, v. 22, p.711-728, 2013.

BALBINOT, R.; SCHUMACHER, M. V.; WATZLAWCIK, L. F.; SANQUETTA, C. R. Inventário do carbono orgânico em um plantio de Pinus taeda aos 5 anos de idade no Rio Grande do Sul. Ciências Exatas e Naturais, Garapuava, v. 5, n. 1, p. 59-68, 2003.

BREIMAN, L. Random Forests. Machine Learning, v. 45, n. 1, p. 5-32, 2001.

CAO, L.; COOPS, N. C.; INNES, J. L.; DAI, J. S.; SHE, G. H. Mapping above- and below ground biomass components in subtropical forests using small-footprint LiDAR. Forests, Basel, v. 5, p.1356- 1373, 2014.

CARVALHO, S. P. C; RODRIGUEZ, L. C. E.; SILVA, L. D.; CARVALHO, L. M. T.; CALEGARIO. N.; LIMA, M. P.; SILVA, C. A.; MENDONÇA, A. R.; NICOLETTI. M.F. Predição do volume de árvores integrando Lidar e Geoestatística. Scientia Forestalis,Piracicaba, v. 43, n. 107, p. 627-637, 2015.

CUTLER, D. R. T. C.; EDWARDS, K. H.; BEARD, A.; CUTLER, K. T.; HESS, J.; LAWLER, J. J. Random forests for classification in ecology. Ecology. V .88, p. 2783-2792, 2007.

CROOKSTON, N. L.; FINLEY, A. Yaimpute: an R package for k-NN imputation. Journal of Statistical Software, Innsbruck, v. 23, p. 1-16, 2008. 
DALLAGNOL, F. S.; MOGNON, F.; SANQUETTA, C. R.; DALLA CORTE, A. P. Teores de carbon em cinco espécies florestais e seus compartimentos. Floresta e Ambiente, Seropédica, v. 18, n. 4, p. 410-416, 2011

EVANS, J. S.; MURPHY, M. A.; HOLDEN, Z. A.; CUSHMAN, S. A. Modeling species distribution and change using Random Forests. In: DREW, C.A.; HUETTMANN, F.; WIERSMA, Y. (Eds.), Predictive Modeling in Landscape Ecology. London: Springer, 2010. p. 139-159.

GARCÍA, M.; RIAÑO, D.; CHUVIECO, E.; MARK DANSON, F. Estimating biomass carbon stocks for a Mediterranean forest in central Spain using LiDAR height and intensity data. Remote Sensing of Environment, New York, v. 114, n. 4, p. 816-830, 2010.

GIONGO, M.; KOEHLER, H. S.; VIDAL, M. R.; BASTOS, P.; SANTOS, A. F.; SANTOPUOLI, G. Estimativa da altura da base das copas com o uso de dados laser scanning aerotransportado (LiDAR). Journal of Biotechnology and Biodiversity, Gurupi, v. 3, n. 3, p. 48-57, 2012.

GIONGO, M.; BASTOS, P. S.; PINTO JÚNIOR, J. P. LIDAR: princípios e aplicações florestais. Pesquisa florestal Brasileira, Brasília, v. 30, n. 63, p. 231-244, 2010.

HUDAK, A. T.; STRAND, E. K.; VIERLING, L. A.; BYRNE, J. C.; EITEL, J. U. H.; MARTINUZZI, S.; FALKOWSKI, M. J. Quantifying aboveground forest carbon pools and fluxes from repeat LiDAR surveys. Remote Sensing Environment, New York, v. 123, n. 1, p. 25-40, 2012.

HYDE, P.; NELSON, R.F.; KIMES, D.; LEVINE. E. Exploring LiDAR - RaDAR Synergy - Predicting aboveground biomass in a southwestern ponderosa pine forest using LiDAR, SAR, and InSAR. Remote Sensing of Environment, New York, v. 106, p. 28-38, 2007.

IBGE - INSTITUTO BRASILEIRO DE GEOGRAFIA E ESTATíSTICA. Produção da Extração Vegetal e da Silvicultura. Rio de Janeiro, v. 29, p.1-56, 2014.

KRAUS, K.; PFEIFER, N. Determination of terrain models in wooded areas with airborne laser scanner data. ISPRS Journal of Photogrammetry and Remote Sensing, v. 53, p. 3193-203, 1998.

LATIFI, H.; NOTHDURFT, A.; KOCH, B. Non-parametric prediction and mapping of standing timber volume and biomass in a temperate forest: application of multiple optical/LiDAR-derived predictors. Forestry, Oxford, v. 83, n.4, p. $395-407,2010$.

LIAW, A.; WIENER, M. Classification and Regression by Random Forest. R News: The Newsletter of the R Project, v. 2, n.3, p. 18-22, 2002.

LU, D.; CHEN, Q.; WANG, G.; MORAN, E.; BATISTELLA, M.; ZHANG, M.; LAURIN, G.V.; SAAH, D. Aboveground forest biomass estimation with Landsat and LiDAR data and uncertainty analysis of the estimates. International Journal of Forestry Research, London, v. 16, 12 p., 2012.

MCGAUGHEY, R. J. FUSION/LDV: Software for LiDAR Data Analysis and Visualization. 3 ed. Seattle: USDA, Forest Service Pacific Northwest Research Station, 2015. 15 p.

MUUKKONEN, P.; MAKIPÄÄ, R. Empirical biomass models of understorey vegetation in boreal forests according to stand and site attributes. Boreal Environment research, Helsinki v.11, p. 355-369. 2006.

MUTANGA, O.; ADAM, E.; CHO, M. A. High density biomass estimation for wetland vegetation using WorldView-2 imagery and random forest regression algorithm. International Journal of Applied Earth Observation and Geoinformation, v. 18, p. 399-406, 2012. 
Silva et al. - Predição da biomassa aérea em plantações de Pinus taeda L. por meio de dados LiDAR aerotransportado

NEUMANN, M.; SAATCHI, S. S.; ULANDER, L. M.; FRANSSON, J. E. Parametric and non-parametric forest biomass estimation from PolInSAR data. In IEEE INTERNATIONAL GEOSCIENCE AND REMOTE SENSING SYMPOSIUM, Vancouver, 2011. Proceedings... New York: IEEE, 2011. p. 420-423.

OLIVEIRA, L. T.; FERREIRA, M. Z.; CARVALHO, L. M. T.; FERRAZ FILHO, A. C.; OlIVEIRA, T. C. A.; SILVEIRA, E. M. O.; ACERBI JUNIOR, F. W. Determinação do volume de madeira em povoamento de eucalipto por escâner a laser aerotransportado., Pesquisa Agropecuária Brasileira Brasília, Brasília, v. 49, n. 9, p. 692-699, 2014.

PENNER, M.; PITT, D. G.; WOODS, M. E. Parametric vs. nonparametric LiDAR models for operational forest inventory in boreal Ontario. Canadian Journal of Remote Sensing, v. 39, n. 5, p. 426-443, 2013.

RIGATTO, A.; DEDECEK, R. A.; MATOS, J. L. M. Influência dos atributos do solo sobre a qualidade da madeira de Pinus taeda para produção de celulose kraft. Scientia Forestalis, Piracicaba, v. 28, n. 2, p. 267-273, 2004.

ROBINSON, A. Equivalence: Provides Tests and Graphics for Assessing Tests of Equivalence, Version 0.7.0, 2015. Disponível em: < https://cran.r-project.org/web/ >. Accesso em 20 Jun. 2017.

SANKEY, T.; SHRESTHA, R.; SANKEY, J. B.; HARDEGREE, S.; STRAND, E. Lidar-derived estimate and uncertainty of carbon sink in successional phases of woody encroachment. Journal of Geophysical Research: Biogeosciences, v. 118, n. 3, p.1144-1155, 2013.

SCHIKOWSKI, A. B.; CORTE, A. P. D.; SANQUETTA, C. R. Modelagem do crescimento e de biomassa individual de Pinus. Pesquisa Florestal Brasileira, Colombo, v. 33, n. 75, p. 269-278. 2013.

SCHUMACHER, M. V.; WITSCHORECK, R.; CALDEIRA, M.V.W.; WATZLAWICK, L.F. Estoque de carbono em florestas de Pinus taeda L. e Acacia mearnsii De Wild plantadas no estado do Rio Grande do Sul-Brasil. In: SANQUETTA, C. R.; et al. (Eds ). As Florestas e o Carbono. Curitiba: UFPR, 2002. p. 141-152.

SETTE JÚNIOR., C. R.; NAKAJIMA, N. Y.; GEROMINI, M. P. Captura de carbono orgânico em povoamentos de Pinus taeda L. na região de Rio Negrinho, SC. Floresta, Curitiba, v. 36, n. 1, p. 33-44, 2006.

SHERIDAN, R. D.; POPESCU, S. C.; GATZIOLIS, D.; MORGAN, C. L. S.; KU, N. W. Modeling Forest Aboveground Biomass and Volume Using Airborne LiDAR Metrics and Forest Inventory and Analysis Data in the Pacific Northwest. Remote Sensing, Basel, v. 7, p. 229-255, 2015.

SILVA, C. A.; KLAUBERG, C.; CARVALHO, S. P. C.; HUDAK, A. T.; RODRIGUEZ, L. C. E. Mapping aboveground carbono stocks using LiDAR data in Eucalyptus spp. Plantations in the state of São Paulo, Brazil. Scientia Forestalis, Piracicaba, v. 42, n. 104, 2014.

SILVEIRA, P. Estimativa da biomassa e carbono acima do solo em um fragmento de Floresta Ombrófila Densa utilizando o método da derivação do volume comercial. Floresta, Curitiba, v. 40, n. 4, p. 789-800, 2010.

SINGH, J.; KNAPP, J. P.; DEMISSIE, M. Hydrologic modeling of the Iroquois River watershed using HSPF and SWAT. ISWS CR 2004-08. Champaign, Illinois State Water Survey. 2004. Disponível em: < www.sws.uiuc. edu/pubdoc/CR/ISWSCR2004-08.pdf >.. Acesso em: 30 maio 2016.

SOMOGYI, Z.; CIENCIALA, E.; MÄKIPÄÄ, R.; LEHTONEN, A.; MUUKKONEN, P. ; WEISS, P. Indirect methods of large-scale forest biomass estimation. European Journal of Forest Research, Berlin, v. 126, n.2, p. 197-207, 2007.

WATZLAWICK, L. F.; CALDEIRA, M. V. W.; VIERA, M.; SCHUMACHER, M.V.; GODINHO, T.O.; BALBINOT, R. Estoque de biomassa e carbono na Floresta Ombrófila Mista Montana, Paraná. Scientia Forestalis, Piracicaba, v. 40, n. 95, p. 345-352, 2012. 
WATZLAWICK, L.F.; KIRCHNER, F.F.; SANGUETTA, C.R. Estimativa de biomassa e carbono em floresta com araucária utilizando imagens do satélite IKONOS II. Ciência Florestal, v. 19, n. 2, p. 169-181, 2009.

WHITE, J. C.; ARNETT, T. T. R.; WULDER, M. A.; TOMPALSKI, P.; COOPS, N. C. Evaluating the impact of leaf-on and leaf-off airborne laser scanning data on the estimation of forest inventory attributes with the areabased approach. Canadian Journal of Forest Research, Ottawa, v. 45, p. 1498-1513. 2015.

ZANDONÁ, D. F.; LINGNAU, C.; NAKAJIMA, N. Y. Varredura a laser aerotransportado para estimativa de variáveis dendrométricas. Scientia Forestalis, Piracicaba, v. 36, p. 295-306, 2008.

ZHAO, F.; GUO, Q.; KELLY, M. Allometric equation choice impacts lidar-based forest biomass estimates: A case study from the Sierra National Forest, CA. Agricultural and Forest Meteorology, v. 165, p. 64-72, 2012.

Recebido em 31/05/2016

Aceito para publicação em 04/05/2017

Sci. For., Piracicaba, v. 45, n. 115, p. 527-539, set. 2017

DOI: dx.doi.org/10.18671/scifor.v45n115.10 
